\title{
Children's use of number line estimation strategies
}

\author{
Dominique Peeters ${ }^{1} \cdot$ Tine Degrande $^{1}$ • \\ Mirjam Ebersbach $^{1,2} \cdot$ Lieven Verschaffel $^{1}$. \\ Koen Luwel ${ }^{1,3}$
}

Received: 24 September 2014 / Revised: 30 December 2014 / Accepted: 5 March 2015

(C) Instituto Superior de Psicologia Aplicada, Lisboa, Portugal and Springer Science+Business Media Dordrecht 2015

\begin{abstract}
This study tested whether second graders use benchmark-based strategies when solving a number line estimation (NLE) task. Participants were assigned to one of three conditions based on the availability of benchmarks provided on the number line. In the bounded condition, number lines were only bounded at both sides by 0 and 200, while the midpoint condition included an additional benchmark at the midpoint and children in the quartile condition were provided with a benchmark at every quartile. First, the inclusion of a midpoint resulted in more accurate estimates around the middle of the number line in the midpoint condition compared to the bounded and, surprisingly, also the quartile condition. Furthermore, the two additional benchmarks in the quartile condition did not yield better estimations around the first and third quartile, because children frequently relied on an erroneous representation of these benchmarks, leading to systematic estimation errors. Second, verbal strategy reports revealed that children in the midpoint condition relied more frequently on the benchmark at the midpoint of the number line compared to the bounded condition, confirming the accuracy data. Finally, the frequency of use of benchmark-based strategies correlated positively with mathematics achievement and tended to correlate positively also with estimation accuracy. In sum, this study is one of the first to provide systematic evidence for children's use of benchmark-based estimation strategies in NLE with natural numbers and its relationship with children's NLE performance.
\end{abstract}

Dominique Peeters and Tine Degrande contributed equally to this work.

Dominique Peeters

dominique.peeters@ppw.kuleuven.be

1 Centre for Instructional Psychology and Technology, KU Leuven, Dekenstraat 2, box 3773, 3000 Leuven, Belgium

2 Institut für Psychologie, Universität Kassel, Holländische Str. 36-38, 34127 Kassel, Germany

3 Centre for Educational Research and Development, KU Leuven - Campus Brussels, Warmoesberg 26, 1000 Brussels, Belgium 
Keywords Number line estimation $\cdot$ Benchmarks $\cdot$ Estimation strategies

Numbers play a prominent role in our daily life. They are used to specify quantities; time; distances; wealth; risks; and many other quantifiable features of objects, people, and situations. Recent studies have shown that the mental representation of numerical magnitudes is related to and predictive for children's mathematics achievement (e.g., Booth and Siegler 2006; Bugden and Ansari 2011; De Smedt et al. 2009; Gilmore et al. 2010; Sasanguie et al. 2012). These findings stress the importance of the ability to understand and process numerical information for success in education and life (Ancker and Kaufman 2007; Finnie and Meng 2001).

During the last decades, the number line estimation (NLE) task became a widely used tool for investigating an individual's mental magnitude representation. In this task, participants are asked to either estimate the spatial position of a number on an empty, physical number line with labeled endpoints (e.g., 0 and 100 or 1000) or, alternatively, estimate the number which corresponds to a given spatial position on such number line. These tasks are known as the number-to-position (NP task) and position-to-number task (PN task), respectively. The pattern that emerges when plotting the estimated positions of the numbers on the number line as a function of their actual position has been assumed to reflect an individual's mental representation of that particular number range (Siegler and Opfer 2003).

Siegler and Opfer (2003) investigated the development of numerical magnitude representations by having a group of second, fourth, and sixth graders as well as adults perform the NLE task on a $0-100$ and $0-1000$ scale. They demonstrated that, with increasing age, participants' NLE patterns evolved from a logarithmic toward a linear pattern on the $0-1000$ scale, whereas all age groups exhibited a linear pattern on the 0-100 scale. This finding suggests that, with increasing age and experience with a specific number range, children's underlying magnitude representation develops from a logarithmically compressed mental number line with increasing numerical magnitudes being successively more closely spaced (Dehaene 1997) toward a linear representation, reflecting the equal spacing principle of the mature number system. Notably, however, some authors have argued that young children's estimation patterns might be described better by a two-segmented linear model than by a logarithmic one (Ebersbach et al. 2008). The steep slope of the first segment refers to the range of numbers that children are familiar with and which they can differentiate easily. Beyond that familiar number range, discrimination between numbers is more difficult, resulting in a second segment with a rather shallow slope. Hence, the change point between the two linear curves functions as an indicator of number familiarity (see also Moeller et al. 2009, for a similar twolinear approach suggesting separate but linear representations for single- and two-digit numbers).

The so-called log-to-lin or representational shift in children's estimation patterns has been replicated at different ages and for different scales (Berteletti et al. 2010; Siegler and Booth 2004; Thompson and Opfer 2010; see Siegler et al. 2009 for a review) as well as in other types of estimation tasks, such as estimating quantities or measurements (Booth and Siegler 2006). Furthermore, the linearity of NLE patterns is strongly related to estimation accuracy (Ashcraft and Moore 2012; Siegler and Booth 2004), performance in basic numerical tasks (Berteletti et al. 2010), and general mathematics achievement as well as other measures of mathematical ability (Ashcraft and Moore 2012; Booth and Siegler 2006, 2008; Schneider et al. 2009; Siegler and Booth 2004). 
Notwithstanding these robust findings, several sources of evidence question whether the NLE task can be considered as a pure measure of an individual's underlying numerical magnitude representation and propose that improvements in NLE performance might be explained - at least to a significant part-by an increasing reliance on a variety of benchmark-based NLE strategies, rather than by changes in children's mental analog of the physical number line per se.

Firstly, by relying on verbal self-reports, Newman and Berger (1984) found that third graders, but not first graders, made use of the midpoint when estimating numbers in the middle range of the number line. Secondly, by closely observing children's overt solution behavior and thus which strategies they use, Petitto (1990) found that the percentage of children using a midpoint strategy increased across grades as arithmetic and counting skills improved. Thirdly, Ashcraft and Moore (2012) observed specific patterns in children's error rates and latencies that deviated strongly from the patterns expected if they would simply be relying on their underlying numerical magnitude representation. More specifically, with age, error rates and latencies first started to decrease at the endpoint of the number line, and later at the midpoint as well, resulting in a typical M-shaped pattern, suggesting the use of an endpoint and a midpoint strategy, respectively (see also White and Szucs 2012). Fourth, Siegler and Opfer (2003) observed that adults' and sixth graders' estimates on a 0-1000 number line were less variable near $0,250,500,750$, and 1000 in comparison to other numbers, suggesting that participants divided the number line into quarters, which were then used as benchmarks to guide their estimates. Fifth, Barth and Paladino (2011) suggested that the typical NLE task should be considered as a proportion judgment task instead of a numerical magnitude estimation task. In their view, estimating the position of a certain number (e.g., 76) on a bounded number line (e.g., 0-100) can be regarded as estimating the size of a part of the line relative to the size of the whole line (i.e., 76/100), which inevitably requires relying on the endpoint of the number line (see e.g., Hollands and Dyre 2000 for a similar explanation of adults' performance in a variety of perceptual proportion tasks). These proportion judgments can be further refined by relying on the midpoint as well, which can be considered as judging part magnitudes relative to a central reference point. Hence, the proportion judgment account suggests that participants make use of benchmarks (i.e., origin, endpoint, and midpoint) to derive the position of the tobe-estimated numbers on a given number line. Making use of such benchmark-based strategies leads to systematic underestimation and overestimation of the to-be-estimated numbers close to the benchmarks (Hollands and Dyre 2000), which can be identified by fitting cyclical power functions on participants' estimation patterns. In line with these results, Barth and Paladino (2011) observed that, with age, children's NLE patterns are successively best described by an unbounded, a one-cycle, and a two-cycle power model (see also Slusser et al. 2013). According to these authors, this development in model complexity reflects children's increasing sophistication in the use of benchmarks on the number line. That is, the unbounded power model characterizes NLE patterns for which only the origin is taken into account. The best fit with a one-cycle model reflects the strategic use of both origin and endpoint, while a best fit with a two-cycle model suggests the reliance on the midpoint too.

Finally, to verify whether the presence of an endpoint indeed leads to estimation patterns that are better fit by a one- or two-cycle power model instead of an unbounded power model, Link et al. (2014) recently tested children from first to fourth grade on both an unbounded (i.e., without an endpoint) and bounded NLE task. Results indicated that, in the unbounded version of the task, children's estimates in all age groups were best fit by an unbounded power model, indicating that they solely relied on the origin of the number line to make their estimates. In the 
bounded number line task, however, children's estimation patterns were, in line with findings of Barth and Paladino (2011), successively best fit by an unbounded, a one-cycle, and a twocycle power model as age increased. Taken together, these studies indicated that as children develop, they start using external (i.e., the endpoint) or self-generated internal (e.g., the midpoint) benchmarks on the number line to guide their estimates.

Although it has become increasingly clear that children appear to use a variety of benchmark-based strategies when making NLEs and that this variation in strategy use accounts for their NLE performance, the precise nature of these strategies has not yet been studied in a direct and systematic way. The goal of the present study was to provide such a direct and systematic analysis, by confronting children with number lines containing different numbers of external benchmarks and by asking them to verbally explain how they solved each item, in addition to an analysis of their error rates. To the best of our knowledge, this is the first study to include such external benchmarks not only at the midpoint but also at the first and third quartile when estimating the position of natural numbers on a number line. Furthermore, while the technique of verbal protocols has already been intensively used in cognitive research on children's mathematical strategies in general (e.g., Siegler and Stern 1998; Torbeyns et al. 2009) and also in one older study on children's NLE (Newman and Berger 1984), the present study is the first wherein young children's verbal strategy reports have been used in a systematic way to help unravel whether and how they rely on external and/or self-generated benchmarks on a number line.

So, in the present study, estimations had to be completed in one of three conditions that differed with respect to the availability of particular benchmarks on a 0 to 200 number line (see Fig. 1). In the bounded condition, number lines were bounded at both sides by the corresponding benchmarks (i.e., 0 and 200). In the midpoint condition, bounded number lines with an additional benchmark at the midpoint (i.e., at 100) were presented. In the quartile condition, children were provided with a bounded number line with a benchmark at every quartile (i.e., at 50, 100, and 150). Three sets of research questions were addressed.

A first set of research questions addressed whether providing additional external benchmarks had a positive effect on children's NLE performance. As mentioned earlier, with increasing age, children have a greater tendency to use an internal benchmark at the midpoint when making NLEs, resulting in an improved NLE performance (e.g., Ashcraft and Moore 2012; Barth and Paladino 2011). Following finding of Siegler and Opfer (2003) that estimates on a $0-1000$ number line were less variable near $0,250,500,750$, and 1000 , which suggested the possible use of internal benchmarks at the quartiles too, we anticipated that providing extra benchmarks at the first and third quartile would further positively affect estimation accuracy. In sum, we expected that an increase in the number of given benchmarks would lead to higher

(a)

(b)

(c)

Fig. 1 Presented number line in a bounded, b midpoint, and $\mathbf{c}$ quartile condition 
overall estimation accuracy. Secondly, the provision of benchmarks was expected to affect the accuracy on the items located near the respective provided benchmarks. More specifically, we predicted that estimations around the first and third quartile would be more accurate in the quartile than in the midpoint and bounded condition, whereas the estimations around the midpoint would be more accurate in the quartile and midpoint than in the bounded condition.

A second set of research questions focused specifically on children's strategy use. Firstly, we expected that an increase in the number of provided benchmarks would result in a greater frequency and variety of reported standard benchmark-based (SBB) strategies (i.e., strategies that made use of self-generated or provided benchmarks that were located at the quartiles of the number line). Secondly, regarding the frequency of these SBB strategies, we expected that children would make more frequently use of strategies that were based on the benchmarks provided in the respective conditions. More specifically, we predicted a relatively high frequency of strategies based on the origin and endpoint of the number line in the bounded condition, whereas in the midpoint condition, we expected more strategies based on the midpoint too, while in the quartile condition, strategies based on the given benchmarks at all quartiles were expected. Thirdly, if children do make use of the benchmarks provided to them, the use of SBB strategies should be reflected in their estimation patterns. Based on previous research (Barth and Paladino 2011; Slusser et al. 2013), we hypothesized that children's estimations in the bounded condition would be best fit by a one-cycle power model, reflecting the strategic use of both the origin and endpoint when making NLEs. Children in the midpoint condition were expected to be best fit by the two-cycle power model since they can rely on the midpoint too, whereas children in the quartile condition should be best fit by a four-cycle power model indicating the use of strategies based on the origin, endpoint, midpoint, first, and third quartile.

As a third and final research question, we investigated the extent to which the use of SBB strategies would be related to children's NLE accuracy, as well as their general mathematics achievement.

\section{Method}

\section{Participants}

Sixty-four second graders ( 34 boys, 30 girls, $M=8.06$ years, $\mathrm{SD}=0.42$ years) were recruited from four classes in two elementary schools located in a rural area of Flanders (Belgium). Most children came from middle-income families. In none of the classes, NLE had been systematically taught. All children participated voluntarily with informed consent of their parents and teachers. Children were told that they could quit the experiment at any moment.

\section{Materials}

Children's NLE performance was assessed by a NP task with a number line labeled at the left end by " 0 " and at the right end by "200." According to the elementary school curriculum and the arithmetic textbook used, second graders had only systematically explored the number range up to 100 at the moment of the data collection (February/March), while the 100-200 range had received no instructional attention at all. To avoid ceiling effects in NLE accuracy and shape of estimation patterns when using a 0-100 number line in second graders (cf. Siegler and Opfer 2003), a 0-200 number line was used. 
For each trial, a new number line with a length of $25 \mathrm{~cm}$ was presented on a separate sheet of paper. To avoid that the presented number might function as an additional benchmark, it was positioned completely on the left of the page. Children had to position 20 randomly chosen numbers on the number line, one from each decade between 0 and 200. These 20 numbers were equally distributed across the number line in order to prevent overestimation of smaller numbers due to oversampling at this end of the number line (Ebersbach et al. 2008). The 20 tobe-positioned numbers were as follows: $5,16,22,38,43,55,62,76,87,91,103,110,129$, $134,146,159,162,173,189$, and 194. The presentation order of these 20 numbers was randomized across participants. Children also received six practice trials, which were also equally spread over the 0 to 200 number range: $4,14,46,105,141$, and 168 . When the estimate of the first or second practice trial was very inaccurate, instructions were shortly repeated. However, at no point, individual feedback was provided.

Children were randomly assigned to one of three conditions (see Fig. 1). Special care was taken to ensure that the number of boys and girls was about equal in the three conditions. In the bounded condition, an empty number line on which the origin and endpoint were indicated by a small vertical line at either side of the number line was presented. The corresponding numbers, 0 and 200, were printed underneath these vertical lines. In the midpoint condition, a benchmark at the middle of the number line was included by presenting a vertical line at the position of 100 but no number. Finally, in the quartile condition, three extra benchmarks were given by introducing vertical lines at positions 50,100, and 150 of the number line. Thus, the benchmarks at 50,100, and 150 in the midpoint and quartile condition were only represented by a vertical line without its corresponding number.

To conclude, children's mathematics achievement was measured by means of the standardized mathematics test of the Flemish Student Monitoring System (Dudal 2000) for the middle of the second grade.

\section{Procedure}

The NLE task was administered individually in a quiet room at the school. Children were given following instructions: "I am going to show you some number lines. These number lines start at 0 and end at 200. For each trial, a number between 0 and 200 is shown on the upper left side of the page. What I want you to do is to put a mark on the line where you think the number would go." The to-be-positioned numbers were read out loud by the experimenter to ensure that children knew which number had to be placed on the number line. Immediately after each trial, the experimenter asked the child to tell how (s)he came to that estimate. In case that the child produced an unclear verbal report, non-intrusive follow-up questions such as "How did you do that?" and "What were you thinking?" were asked. At the end of the task, children in the midpoint and quartile condition were asked to indicate which number corresponded to the vertical line at 100 (midpoint condition) or 50, 100, and 150 (quartile condition). The NLE task lasted 30 to 60 min per child and was completely recorded with a voice recorder.

\section{Strategy classification scheme}

Strategy reports were analyzed through a self-designed classification scheme consisting of three main categories: perceptual strategies, SBB strategies, and non-SBB strategies (see Fig. 2). Perceptual strategies include seeing, guessing, and knowing (Gandini et al. 2010; Gandini et al. 2008). SBB and non-SBB strategies refer to strategies that make use of a 
benchmark. Specifically, SBB strategies make use of self-generated or externally provided benchmarks that are located at the quartiles on the number line: origin $(0 \%)$, first quartile (25\%), midpoint (50\%), third quartile (75\%), and endpoint (100\%). For instance, when a child had to locate 44 on the $0-200$ number line and reported that (s)he had first looked at the benchmark representing 50 and then located 44 a bit to the left of that benchmark, it was coded as $25 \%{ }^{1}$ Non-SBB strategies are based on other, self-generated benchmarks different from those five standard benchmarks, such as a benchmark corresponding to an answer to a previous item. Finally, a rest category was available for cases that were not classifiable in the above-mentioned categories.

Reliability of the classification scheme was assessed by testing the agreement in classification of two independent raters who classified all experimental trials of eight randomly chosen participants by means of Cohen's kappa. This inter-rater reliability measure was 0.89 , indicating almost perfect agreement according to the standards of Landis and Koch (1977).

\section{Results}

\section{Estimation accuracy}

For each of the three conditions, individual estimates that deviated more than 2 SD from children's mean estimate for the to-be-positioned number were excluded. In total, 71 estimates out of a total of 1280 were removed $(5.5 \%)$. These 71 estimates were similarly distributed across conditions. Estimation accuracy was measured in terms of percent absolute error (PAE). Tukey's HSD tests were used in all post hoc comparisons.

Overall estimation accuracy A one-way ANOVA assessing the effect of condition (bounded, midpoint, quartile) on overall PAE was significant, $F(2,61)=16.66, p<0.0001$. Estimates in the quartile condition $(M=14.88, \mathrm{SD}=5.26)$ were, contrary to our predictions, significantly less accurate than in the midpoint $(M=7.32, \mathrm{SD}=4.39, p<0.0001)$ and even the bounded condition $(M=9.66, \mathrm{SD}=5.26, p=0.0007)$, while there was no significant difference between the latter two conditions. So, the provision of additional benchmarks in the quartile condition seemed to be counterproductive instead of helpful for the overall accuracy of children's NLEs.

Estimation accuracy near the benchmarks To get a closer view on the accuracy near the location of the standard benchmarks, we conducted a contour analysis on the trials where SBB strategies were used (Ashcraft and Moore 2012). Therefore, we averaged on a child-by-child basis the observed PAEs for the two items located immediately before and after the midpoint (i.e., 91 and 103) and quartiles (i.e., 43 and 55; 146 and 159). For the origin and endpoint, our measure was based on the PAE of the number immediately after the origin and before the endpoint, respectively (i.e., 5 and 194). A 3 (condition: bounded, midpoint, quartile) $\times 5$ (location $0,25,50,75,100 \%$ ) ANOVA with repeated measures on the last variable and the PAEs as dependent variable revealed a significant main effect of condition $(F(2,61)=19.47$, $p<0.0001)$.

\footnotetext{
${ }^{1}$ We coded the benchmarks as represented by the child even when misrepresented (e.g., when they thought the $25 \%$ benchmark represented 100 instead of 50 ).
} 


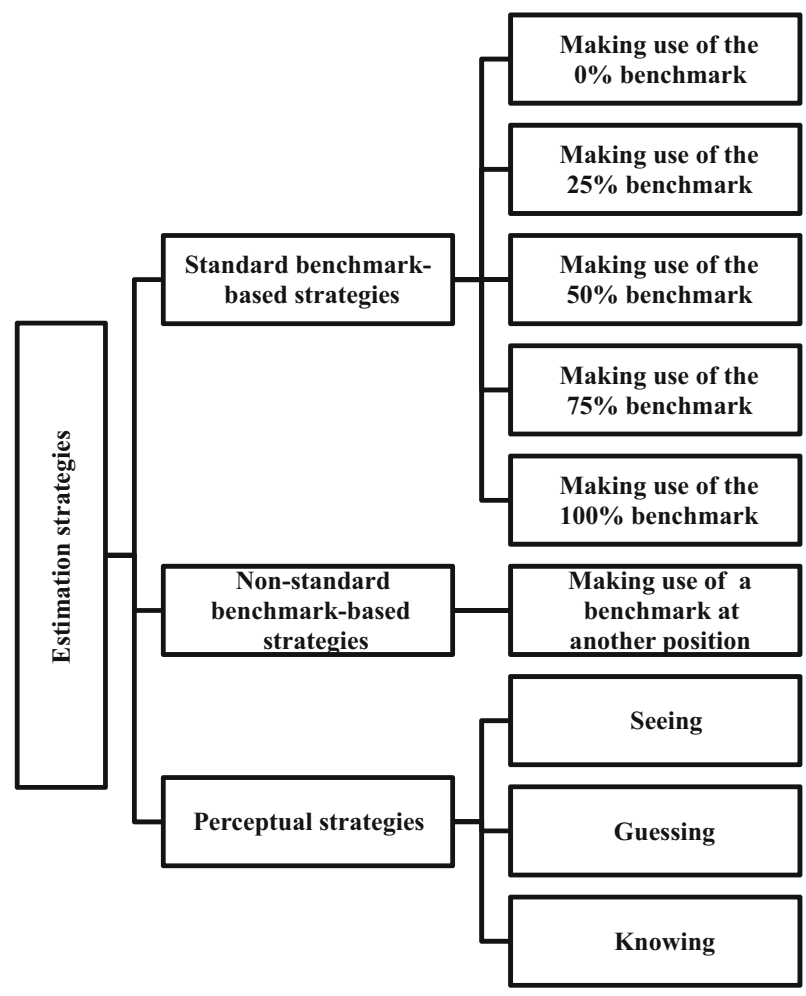

Fig. 2 Decision tree for the classification of NLE strategies

Furthermore, a significant interaction effect between condition and location was observed $(F(8,244)=5.32, p<0.0001)$ (see Fig. 3). No significant difference between conditions at the origin, third quartile, and endpoint was found. However, at the first quartile, estimates in the quartile condition $(M=15.35, \mathrm{SD}=9.18)$ were significantly less accurate than estimates in the midpoint condition $(M=6.80, \mathrm{SD}=5.32, p<0.0001)$, which is opposite to our expectation. At the midpoint, estimates in the midpoint condition $(M=6.95, \mathrm{SD}=8.60)$ were significantly more accurate than in the bounded $(M=14.89, \mathrm{SD}=8.33, p=0.0004)$ and even the quartile condition $(M=19.19, \mathrm{SD}=9.00, p<0.0001)$. Thus, the inclusion of a midpoint did result in more accurate estimates around the middle of the number line in the midpoint condition but not in the quartile condition. Furthermore, the two additional benchmarks in the quartile condition did not lead to better estimates around first and third quartile in that condition.

Nature of the estimation errors In search of an explanation for the remarkable finding that estimation performance around the benchmarks was not better in the quartile condition compared to the bounded condition, we analyzed the estimation data of this condition in greater detail.

First, we looked at the items at 25, 50, and $75 \%$ of the number line. A closer inspection of children's estimated positions revealed that children from the quartile condition gave estimates that deviated systematically from the correct answer. More specifically, estimates seemed to follow logically from an erroneous interpretation of the external benchmarks provided in this 


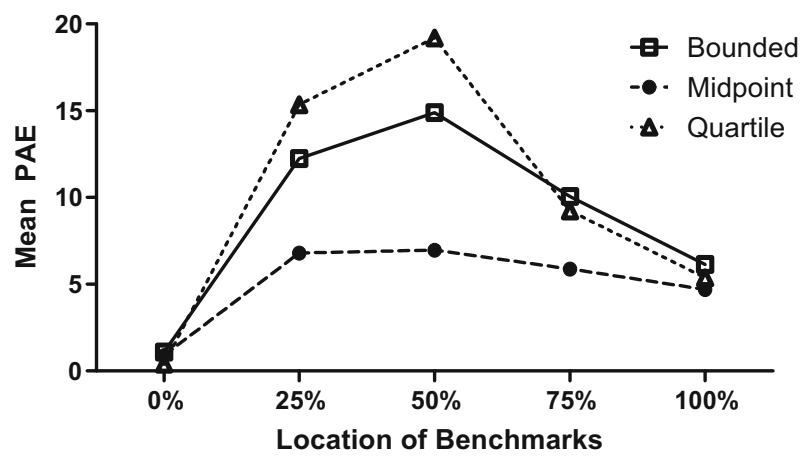

Fig. 3 Mean percentage of absolute error (PAE) around the standard benchmarks as a function of condition

condition. For example, a child estimated 103 as 153 because (s)he seems to have misallocated (the number) 100 at the $75 \%$ benchmark (i.e., the number 150). In an attempt to identify the trials on which such erroneously identified benchmarks might have been used, we calculated for each to-be-positioned number two intervals by adding and subtracting the value of the respective (misrepresented) benchmarks plus or minus ten to/from the to-be-positioned number. For instance, if a child had to estimate the position of 103 , we assumed that his/her estimate would fall in the interval (43-63) if (s)he erroneously represented the benchmark at $25 \%$ as 100 and in the interval (143-163) if (s)he erroneously represented the benchmark at $75 \%$ as 100 . Identification of these systematic errors revealed that $16 \%$ of the estimates around the first quartile, such as 43 and 55, were due to a misinterpretation of this first quartile as the midpoint (see Table 1). For the estimates around the midpoint, such as 91 and 103, results indicated that $39 \%$ of the estimates appeared to be the result of an incorrect allocation of the number 100 on the position of 150 (i.e., the third quartile). Also, $10 \%$ of the estimates around the midpoint in the quartile condition were based on the $25 \%$ benchmark. Remarkably, none of the estimates around the third quartile, such as 146 and 159, was due to incorrectly interpreting the first quartile or midpoint as the third quartile. Hence, this analysis suggested that a substantial number of children from the quartile condition associated the three given benchmarks with a wrong number to guide their estimates.

Secondly, we looked at children's responses to the general question at the end of the interview, concerning what number was represented by the benchmarks located at 25, 50, and

Table 1 Percentage of estimates around the three benchmarks based on wrongly used benchmarks for the quartile condition

\begin{tabular}{llll}
\hline \multirow{4}{*}{ Benchmark at } & \multicolumn{2}{l}{ Wrongly interpreted as } \\
\cline { 2 - 4 } & $25 \%$ & $50 \%$ & $75 \%$ \\
\hline $25 \%$ & & $16 \%$ & $2 \%$ \\
& & $(n=7)$ & $(n=1)$ \\
$70 \%$ & $10 \%$ & & $39 \%$ \\
$75 \%$ & $(n=4)$ & $0 \%$ & $(n=16)$ \\
\hline
\end{tabular}

For instance, $39 \%$ of the trials surrounding the $50 \%$ benchmark (i.e., 100) were estimated around the $75 \%$ benchmark due to wrongly locating the number 100 on the position of 150 (i.e., $75 \%$ benchmark) 
$75 \%$ of the number line (see Table 2). In the midpoint condition, $33 \%$ of the children referred to a number different from 100 when being asked which number was represented by the $50 \%$ benchmark. In the quartile condition, $86 \%$ of the children wrongly identified both the vertical line at 25 and $50 \%$, and even $90 \%$ misinterpreted the third quartile. Interestingly, of this $90 \%$, about half of them (i.e., $47 \%$ ) identified the third quartile as representing 100 instead of 150 .

\section{Strategy use}

Taking into account the unexpected finding that so many children from the quartile condition were unable to identify and properly use the value of the externally provided benchmarks, we decided to exclude the data from the quartile condition from the remainder of the analyses.

Frequency of strategy use We analyzed how often children made use of a strategy from one of the three main strategy categories in our classification scheme and whether this varied as a function of condition. We therefore conducted a 2 (condition: bounded vs. midpoint) $\times 3$ (strategy category: perceptual strategies, SBB strategies, non-SBB strategies) ANOVA with repeated measures on the last variable and the percentage of trials being solved with a specific strategy as dependent variable. This ANOVA revealed a significant main effect of strategy category, $F(2,80)=32.58, p<0.0001$. Overall, the SBB strategies $(M=55 \%, \mathrm{SD}=26 \%)$ were used most frequently, followed by the non-SBB strategies $(M=30 \%, \mathrm{SD}=23 \%)$ and the perceptual strategies $(M=12 \%, \mathrm{SD}=12 \%$, all $p \mathrm{~s}<0.01)$.

Frequency of SBB strategies We examined whether the use of the distinct SBB strategies differed as a function of condition and number range. Since the mean frequency of use of the 25 and the $75 \%$ SBB strategies across the bounded and midpoint condition was only 4 and $2 \%$, respectively, we decided to exclude these strategies from the remainder of the analyses. A 2 (condition: bounded vs. midpoint) $\times 3$ (SBB strategy $0,50,100 \%) \times 4$ (number range $0-50$, 50-100, 100-150, 150-200) ANOVA with repeated measures on the last two variables was conducted on the frequency of strategy use. A significant main effect of strategy, $F(2,80)=$ $13.17, p<0.0001$, was found. We further observed a significant SBB strategy $\times$ condition interaction, $F(2,80)=10.35, p=0.0001$, indicating that the $50 \%$ strategy was used more in the midpoint condition $(M=30 \%, \mathrm{SD}=29 \%)$ compared to the bounded condition $(M=15 \%$, $\mathrm{SD}=21 \%, p=0.001)$. Also, a significant $\mathrm{SBB}$ strategy $\times$ number range interaction was found, $F(6,240)=36.59, p<0.0001$ (see Fig. 4). We found that, in range $0-50$, the $0 \%$ strategy $(M=$ $29 \%, \mathrm{SD}=25 \%)$ was used significantly more frequently than the $50 \%(M=3 \%, \mathrm{SD}=9 \%)$

Table 2 Percentage of wrongly identified benchmarks for the midpoint and quartile condition based on interview data

\begin{tabular}{llll}
\hline & \multicolumn{2}{l}{ Benchmarks at } & \\
\cline { 2 - 4 } Condition & $25 \%$ & $50 \%$ & $75 \%$ \\
\hline Midpoint & & $33 \%$ & \\
& & $(n=7)$ & $91 \%$ \\
Quartile & $86 \%$ & $86 \%$ & $(n=19)$ \\
& $(n=18)$ & $(n=18)$ & \\
\hline
\end{tabular}




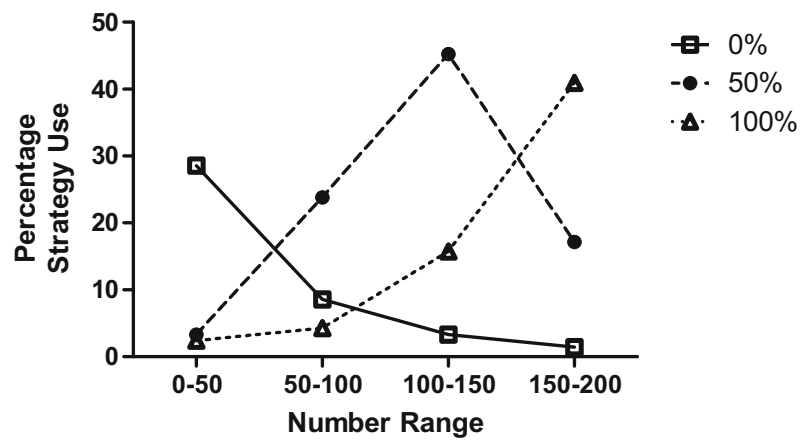

Fig. 4 Percentage use of the 0, 50, and $100 \%$ SBB strategies for each range

and $100 \%(M=2 \%, \mathrm{SD}=11 \%)$ strategy (both $p \mathrm{~s}<0.0001)$. In range 50-100 and 100-150, the $50 \%$ strategy $(M=24 \%, \mathrm{SD}=22 \%$ and $M=45 \%, \mathrm{SD}=29 \%$, respectively) was used significantly more frequently than the $0 \%(M=9 \%, \mathrm{SD}=17 \%$ and $M=3 \%, \mathrm{SD}=11 \%$, respectively) and $100 \%(M=4 \%, \mathrm{SD}=11 \%$ and $M=16 \%, \mathrm{SD}=22 \%$, respectively) strategy (all $p \mathrm{~s}<0.05)$. Finally, in the range, $150-200$, the $100 \%$ strategy $(M=41 \%$, SD=30\%) was used significantly more often than the $0 \%(M=1 \%, \mathrm{SD}=9 \%)$ and $50 \%(M=17 \%, \mathrm{SD}=$ $22 \%$ ) strategy (both $p$ s $<0.0001$ ). Also, the $50 \%$ strategy was used more often than the $0 \%$ strategy $(p<0.05)$. The three-way interaction was not significant. To summarize, the $50 \%$ strategy was used more in the midpoint condition in comparison to the bounded condition. Moreover, children specifically relied on the origin and endpoint (and to a lesser extent also on the midpoint) in the outer ranges, whereas they especially used the $50 \%$ benchmark or midpoint in the two middle ranges.

Model fittings To test whether the use of SBB strategies was reflected in children's estimation patterns, analyses of individual estimation patterns were conducted. We separately fitted an unbounded, a one-cycle, and a two-cycle power model on the estimates of each child in the bounded and midpoint condition. The unbounded power model was also fitted to identify children who only used the origin to guide their estimates. Since the 25 and $75 \%$ strategies were used very infrequently (i.e., altogether on only $6 \%$ of all trials) and since we had left out the quartile condition in all analyses concerning strategy use, a four-cycle power model was not fitted on the data. The Akaike information criterion corrected for small samples (AICc) was used (as in Barth and Paladino 2011; Slusser et al. 2013) to determine which model could best explain children's estimation patterns. This measure takes into account goodness of fit and model complexity (i.e., number of parameters) whereby a lower AICc value refers to a better model fit. Differences in AICc scores (i.e., $\triangle \mathrm{AICc}$ ) reflect the amount of support for one specific model in comparison to the other models. According to Burnham and Anderson (2002), models having a $\Delta$ AICc within 0-2 of the best model have substantial support and should be taken into consideration when making inferences, models with a $\triangle \mathrm{AICc}$ within 4-7 have considerably less support, and models with a $\Delta \mathrm{AICc}>10$ have essentially no support. Most children in the bounded condition, namely $67 \%$,were best fit by an unbounded power model, followed by 14 and $19 \%$ for the one-cycle and two-cycle model, respectively (see Table 3). In the midpoint condition, 48, 14, and $38 \%$ of the children were best fit by an unbounded, a one-cycle, and a two-cycle model, respectively. Even though we observed a decrease in the percentage of children being best fit by the unbounded power model and an 
Table 3 Percentage of children best fit by each power model and the difference scores for AICc in the bounded and midpoint condition

\begin{tabular}{|c|c|c|c|c|}
\hline \multirow[b]{2}{*}{ Power models } & \multicolumn{2}{|c|}{ Bounded condition } & \multicolumn{2}{|c|}{ Midpoint condition } \\
\hline & $\%$ & $\triangle \mathrm{AICc}$ & $\%$ & $\triangle \mathrm{AICc}$ \\
\hline \multirow[t]{2}{*}{ Unbounded } & 67 & One-cycle 11.96 & 48 & One-cycle 10.58 \\
\hline & $(n=14)$ & Two-cycle 15.87 & $(n=10)$ & Two-cycle 13.00 \\
\hline \multirow[t]{2}{*}{ One-cycle } & 14 & Unbounded 3.56 & 14 & Unbounded 2.96 \\
\hline & $(n=3)$ & Two-cycle 9.63 & $(n=3)$ & Two-cycle 2.19 \\
\hline \multirow[t]{2}{*}{ Two-cycle } & 19 & Unbounded 5.95 & 38 & Unbounded 9.70 \\
\hline & $(n=4)$ & One-cycle 5.00 & $(n=8)$ & One-cycle 9.48 \\
\hline
\end{tabular}

increase in the percentage of children being best fit by the two-cycle model from the bounded to the midpoint condition, a chi-square test failed to reveal a significant association between condition and best model fit $\left(\chi^{2}(2, N=42)=2.00, p>0.10\right)$.

\section{Relationship between SBB strategy use and children's NLE performance and math} achievement To investigate the extent to which the use of SBB strategies was related to children's NLE accuracy, as well as their general mathematics achievement, we calculated the correlations between PAE, mathematics achievement, and the use of SBB strategies (see Table 4). The use of SBB strategies correlated significantly with mathematics achievement $(r=0.40, p<0.01)$ and tended to correlate negatively with PAE $(r=-0.28, p=0.078)$ and thus positively with the accuracy of children's NLEs.

\section{Discussion}

Both older and more recent sources of indirect evidence suggest that, when making NLEs, children make use of strategies based on given or self-generated benchmarks (Ashcraft and Moore 2012; Barth and Paladino 2011; Ebersbach et al. 2013; Newman and Berger 1984; Petitto 1990; Siegler and Opfer 2003; White and Szucs 2012). The purpose of the present study was to perform a direct and systematic analysis of children's strategy use by asking second-grade children to verbally explain how they solved each NLE item, in addition to analyzing their error rates. To achieve this goal, second graders had to solve a NP task in which the number of externally provided benchmarks was manipulated in three separate conditions: a bounded, a midpoint, and a quartile condition.

Table 4 Correlations between NLE accuracy, mathematics achievement, and SBB strategy use

1.

1. NLE accuracy (PAE)

2. Mathematics achievement $\quad-0.12$

3. \% Total SBB strategy use

$-0.28$

$0.40^{*}$

$* p<0.01$ 
Firstly, we examined the effect of given benchmarks on children's NLE performance. The underlying idea was that, if the application of benchmark-based strategies indeed mediates children's NLE performance, then providing them with more benchmarks should lead to more frequent and more efficient strategic behavior based on these benchmarks and, consequently, to higher performance on the NLE task in terms of estimation accuracy. However, a larger number of provided benchmarks did not have the expected positive effect on children's overall estimation accuracy. Also, we performed a contour analysis (Ashcraft and Moore 2012), which revealed that, as hypothesized, the provision of a benchmark at the midpoint did lead to more accurate estimates for items around the middle of the number line compared to the bounded condition but - again unexpectedly — also compared to the quartile condition. The two additional benchmarks in the quartile condition, however, did not lead to better estimates around first and third quartile in that condition. In search of an explanation for the unexpected findings for the quartile condition, we performed two additional analyses, which jointly provided strong evidence that the use of erroneously represented benchmarks led to systematic NLE errors. More specifically, children in this condition often made erroneous associations between the benchmark numbers 50,100, and 150, on the one hand, and their corresponding location on the external number line, on the other hand.

Secondly, children's strategy use in the bounded and midpoint condition was investigated. We looked whether a larger number of provided benchmarks led to an increase in the number of strategies that were based on self-generated benchmarks or on benchmarks that were externally provided at 0,50 , or $100 \%$ of the number line. As expected, the $50 \%$ strategy was used more frequently in the midpoint than in the bounded condition. Furthermore, we observed the expected associations between type of SBB strategy and number range, in the sense that each type of SBB strategy was used mostly in the number range(s) where one would rationally expect it. For instance, the $0 \%$ strategy was used most frequently for estimating numbers near the origin, whereas the 50 and $100 \%$ strategy were used most frequently for estimations near the midpoint and endpoint, respectively.

Furthermore, we hypothesized that the use of these benchmarks should be reflected in children's estimation patterns. By separately fitting an unbounded, a one-cycle, and a twocycle power model on the estimates of each child in the bounded and midpoint condition, we revealed that the percentage of children being best fit by the two-cycle power model increased from the bounded (i.e., $19 \%$ ) to the midpoint (i.e., $38 \%$ ) condition. This result is in line with the verbal reports on children's strategy use indicating a more frequent use of the $50 \%$ strategy in the midpoint than in the bounded condition. However, we also observed a large percentage of children in the bounded and midpoint condition who were fitted best by an unbounded power model. A possible explanation might be that the unfamiliar number range used in the present study (i.e., 0-200) prevented the strategic use of any other benchmark beyond the origin.

Finally, the extent to which the use of SBB strategies was related to children's NLE accuracy and their general mathematics achievement was investigated. We found a significant correlation between the use of SBB strategies and mathematics achievement and a marginally significant correlation between the use of SBB strategies and estimation accuracy. These findings suggest that children who are more proficient in mathematics make greater use of SBB strategies for making NLEs. Moreover, the use of these SBB strategies seems to lead to more accurate estimates.

Our findings as summarized above have implications for theory, research, and educational practice. 
From a theoretical perspective, our findings, first of all, involve a plea for taking into account more seriously participants' strategy use when solving the NLE task (Barth and Paladino 2011; Ebersbach et al. 2013; Link et al. 2014; Slusser et al. 2013). The reported evidence that the provision of an (unlabeled) benchmark at the midpoint affects children's NLE accuracy raises doubt whether the NLE task can be considered as a pure measure of one's underlying magnitude representation. However, we also observed that additional (unlabeled) benchmarks at 25 and $75 \%$ may have no effect or even a detrimental effect. A convincing explanation for this finding is still lacking, but two related post hoc analyses suggested that this might be attributed to an erroneous determination of these extra benchmarks. This raises the question why children from the quartile condition departed from wrong associations between the numbers of 50,100, and 150 and their location on the number line. Most probably, this wrong association might be caused by children's lacking familiarity with these numbers (Ebersbach et al. 2008), their inability to reason proportionally (Boyer et al. 2008) and/or to technically execute the required multiplicative operations in the proportional relation (Barth et al. 2009). For instance, Boyer et al. (2008) showed that 10 to 12 years olds have difficulty solving proportional reasoning problems when the proportions are represented in discrete quantities. Furthermore, it could be argued that children's familiarity with the base 10 system would lead to better estimations with benchmarks representing decades (i.e., 10, 20, ...) rather than larger quantities such as multiples of 50. A possible way to further investigate this issue would be to compare children's NLE performance and strategy use when confronted with a bounded number line, a number line with a benchmark at each decile, and a number line with benchmarks at each quartile.

Interestingly, Siegler and Thompson (2014) found that not all types of benchmarks had a beneficial effect on fifth graders' performance when estimating the position of common fractions on a number line. More specifically, external benchmarks that divided a 0-1 number line into tenths, quarters, or fifths led to less accurate estimates than having no benchmark or a midpoint benchmark. It was demonstrated that the decile, quartile, and quintile benchmarks led to an improper encoding of the fractions on the basis of its numerator or denominator rather than on the fraction magnitude, which in its turn led to less accurate estimates. Obviously, this explanation cannot account for the present findings since all the presented numbers in our study were whole numbers, and thus, children could not encode the presented numbers in terms of numerator and denominator but only as a magnitude.

From a methodological perspective, we point out that we tried investigating children's strategies for NLE by collecting verbal reports. The actual distribution of the different SBB strategies across the different ranges of the number line provides additional support for the validity of these reports. However, asking children to provide a verbal self-report after each trial might influence their subsequent problem-solving behavior, an issue known as reactivity (Russo et al. 1989). This reactivity is limited when participants' strategies can be retrieved from short-term memory without competing for attentional resources. Since we asked children to verbalize their strategies immediately after each trial when the number line and their given estimate were still available to them, we are confident that reactivity was not an issue. We, nevertheless, suggest to also collect other kinds of data on NLE strategies such as (videobased) observations of children's actual solution behavior, their pointing behavior on the number line (for instance, when presented on a tablet; Vermeulen et al. 2015), and/or their eye movements (Schneider et al. 2009).

We finally turn to some implications for early and elementary mathematics education. First, our research suggests that it is possible to improve the accuracy of children's NLEs by 
providing them external benchmarks, although our study also yielded strong evidence that this instructional intervention may also lead to unexpectedly negative results, particularly for benchmarks that go beyond the $50 \%$ benchmark and when working in an unfamiliar number domain. Anyhow, when adding such extra benchmarks, it is recommendable to check carefully if learners interpret and represent them properly. More generally, our study suggests that it would be interesting to design and evaluate instructional environments that stimulate the development of children's NLEs. Arguably, several such environments have already been developed, typically in a game-based environment (Ramani et al. 2012; Whyte and Bull 2008) and some of these studies have already yielded promising results. However, to the best of our knowledge, so far, no such intervention has paid pivotal attention at the development of children's estimation strategies by working intentionally and systematically at children's underlying (benchmark-based) strategies. However, it should be noted that, given the results of the present study, these interventions might improve children's NLE performance, but this improvement does not necessarily entail an improvement in children's underlying magnitude representation.

Acknowledgments The conduct of this study was supported by grant GOA 2012/010 of the Research Fund KU Leuven, Belgium, and by grant DFG: EB462/1-1 of the German Research Foundation to the fourth author.

\section{References}

Ancker, J. S., \& Kaufman, D. (2007). Rethinking health numeracy: a multidisciplinary literature review. Journal of the American Medical Informatics Association, 14, 713-721.

Ashcraft, M. H., \& Moore, A. M. (2012). Cognitive processes of numerical estimation in children. Journal of Experimental Child Psychology, 111, 246-267.

Barth, H. C., \& Paladino, A. M. (2011). The development of numerical estimation: evidence against a representational shift. Developmental Science, 14, 125-135.

Barth, H. C., Baron, A., Spelke, E., \& Carey, S. (2009). Children's multiplicative transformations of discrete and continuous quantities. Journal of Experimental Child Psychology, 103, 441-454.

Berteletti, I., Lucangeli, D., Piazza, M., Dehaene, S., \& Zorzi, M. (2010). Numerical estimation in preschoolers. Developmental Psychology, 46, 545-551.

Booth, J. L., \& Siegler, R. S. (2006). Developmental and individual differences in pure numerical estimation. Developmental Psychology, 41, 189-201.

Booth, J. L., \& Siegler, R. S. (2008). Numerical magnitude representations influence arithmetic learning. Child Development, 79, 1016-1031.

Boyer, T. W., Levine, S. C., \& Huttenlocher, J. (2008). Development of proportional reasoning: where young children go wrong. Developmental Psychology, 44, 1478-1490.

Bugden, S., \& Ansari, D. (2011). Individual differences in children's mathematical competence are related to the intentional but not automatic processing of Arabic numerals. Cognition, 118, 32-44.

Burnham, K. P., \& Anderson, D. R. (2002). Model selection and multimodel inference: a practical informationtheoretic approach (2nd ed.). New York: Springer.

De Smedt, B., Verschaffel, L., \& Ghesquière, P. (2009). The predictive value of numerical magnitude comparison for individual differences in mathematics achievement. Journal of Experimental Child Psychology, 103, 469-479.

Dehaene, S. (1997). The number sense. Oxford: Oxford University Press.

Dudal, P. (2000). Leerlingvolgsysteem: Wiskunde-Toetsen 1-2-3. Basisboek [Student monitoring system: Mathematics - tests 1-2-3 manual]. Leuven: Garant.

Ebersbach, M., Luwel, K., Frick, A., Onghena, P., \& Verschaffel, L. (2008). The relationship between the shape of the mental number line and familiarity with numbers in 5- to 9-year old children: evidence for a segmented linear model. Journal of Experimental Child Psychology, 99, 1-17.

Ebersbach, M., Luwel, K., \& Verschaffel, L. (2013). Comparing apples and pears in studies on magnitude estimates. Frontiers in Cognitive Science, 4, 1-6.

Finnie, R., \& Meng, R. (2001). Cognitive skills and the youth labour market. Applied Economics Letters, 8, 675-679. 
Gandini, D., Lemaire, P., \& Dufau, S. (2008). Older and young adults'strategies in approximative quantification. Acta Psychologica, 129, 175-189.

Gandini, D., Ardiale, E., \& Lemaire, P. (2010). Children' strategies in approximate quantification. Current Psychology Letters: Behaviour, Brain, \& Cognition, 26, 1-14.

Gilmore, C. K., McCarthy, S. E., \& Spelke, E. S. (2010). Non-symbolic arithmetic abilities and mathematics achievement in the first year of formal schooling. Cognition, 115, 394-406.

Hollands, J. G., \& Dyre, B. (2000). Bias in proportion judgments: the cyclical power model. Psychological Review, 107, 500-524.

Landis, J. R., \& Koch, G. G. (1977). The measurement of observer agreement for categorical data. Biometrics, $33,159-174$.

Link, T., Huber, S., Nuerk, H.-C., \& Moeller, K. (2014). Unbounding the mental number line- new evidence on children's spatial representation of numbers. Frontiers in Psychology, 4, 1-12.

Moeller, K., Pixner, S., Kaufmann, L., \& Nuerk, H.-C. (2009). Children's early mental number line: logarithmic or decomposed linear? Journal of Experimental Child Psychology, 103, 503-515.

Newman, R. S., \& Berger, C. F. (1984). Children's numerical estimation: flexibility in the use of counting. Journal of Educational Psychology, 76, 55-64.

Petitto, A. L. (1990). Development of number line and measurement concepts. Cognition and Instruction, 7, 5578.

Ramani, G. B., Siegler, R. S., \& Hitti, A. (2012). Taking it to the classroom: number board games as a small group learning activity. Journal of Educational Psychology, 104, 661-672.

Russo, J. E., Johnson, E. J., \& Stephens, D. L. (1989). The validity of verbal protocols. Memory and Cognition, $17,759-769$.

Sasanguie, D., De Smedt, B., Defever, E., \& Reynvoet, B. (2012). Association between basic numerical abilities and mathematics achievement. British Journal of Developmental Psychology, 30, 344-357.

Schneider, M., Grabner, R. H., \& Paetsch, J. (2009). Mental number line, number line estimation, and mathematical school achievement: their interrelations in grades 5 and 6. Journal of Educational Psychology, 101, 359-372.

Siegler, R. S., \& Booth, J. L. (2004). Development of numerical estimation in young children. Child Development, 75, 428-444.

Siegler, R. S., \& Opfer, J. E. (2003). The development of numerical estimation: evidence for multiple representations of numerical quantity. Psychological Science, 14, 237-243.

Siegler, R. S., \& Stern, E. (1998). Conscious and unconscious strategy discoveries: a microgenetic analysis. Journal of Experimental Psychology: General, 127, 377-397.

Siegler, R. S., \& Thompson, C. A. (2014). Numerical landmarks are useful—except when they're not. Journal of Experimental Child Psychology, 120, 39-58.

Siegler, R. S., Thompson, C. A., \& Opfer, J. E. (2009). The logarithmic-to-linear shift: one learning sequence, many tasks, many time scales. Mind, Brain, and Education, 3, 143-150.

Slusser, E. B., Santiago, R. T., \& Barth, H. C. (2013). Developmental change in numerical estimation. Journal of Experimental Psychology: General, 142, 193-208.

Thompson, C. A., \& Opfer, J. E. (2010). How 15 hundred is like 15 cherries: effect of progressive alignment on representational changes in numerical cognition. Child Development, 81, 1768-1786.

Torbeyns, J., De Smedt, B., Ghesquière, P., \& Verschaffel, L. (2009). Acquisition and use of shortcut strategies by traditionally schooled children. Educational Studies in Mathematics, 71, 1-17.

Vermeulen, J. A., Scheltens, F., \& Eggen, T. J. H. M., (2015). Strategie-identificatie met de lege getallenlijn: Een vergelijking tussen tablet en papier [Strategy identification on the empty number line: a comparison between tablets and paper]. Pedagogische Studiën, 92, 39-54.

White, S. L. J., \& Szucs, D. (2012). Representational change and strategy use in children's number line estimation during the first years of primary school. Behavioral and Brain Functions, 8, 1-12.

Whyte, J. C., \& Bull, R. (2008). Number games, magnitude representation, and basic number skills in preschoolers. Developmental Psychology, 44, 588-596.

Dominique Peeters. Centre for Instructional Psychology and Technology, KU Leuven, Dekenstraat 2, box 3773, 3000 Leuven, Belgium. E-mail: dominique.peeters@ppw.kuleuven.be

Current themes of research:

Number line estimation. Numerical cognition. Strategy development. 
Tine Degrande. Tine Degrande. Centre for Instructional Psychology and Technology, KU Leuven, Dekenstraat 2, box 3773, 3000 Leuven, Belgium.

Current themes of research:

Proportional reasoning. Additive reasoning. Cognitive development. Mathematics. Primary education.

Most relevant publications in the field of Psychology of Education:

Degrande, T., Verschaffel, L., Van Dooren, W. (2014). How do Flemish children solve 'Greek' wordproblems? On children's quantitative analogical reasoning in mathematically neutral word problems. Mediterranean Journal for Research in Mathematics Education, 13(1-2): 57-74.

Mirjam Ebersbach. Centre for Instructional Psychology and Technology, KU Leuven, Dekenstraat 2, box 3773, 3000 Leuven, Belgium; Institut für Psychologie, Universität Kassel, Holländische Str. 36-38, 34127 Kassel, Germany.

Current themes of research:

Cognitive development. Development of information processing. Mathematical knowledge. Implicit and explicit knowledge. Embodiment.

Most relevant publications in the field of Psychology of Education:

Ebersbach, M., \& Erz, P. (2014). Symbolic versus non-symbolic magnitude estimations among children and adults. Journal of Experimental Child Development, 128, 52-68. doi:10.1016/j.jecp.2014.06.005.

Ebersbach, M., Luwel, K., \& Verschaffel, L. (2014). Further evidence for a spatial-numerical association in children before formal schooling. Experimental Psychology, 18, 1-7. doi:10.1027/1618-3169/a000250.

Ebersbach, M., Luwel, K., Frick, A., Onghena, P., \& Verschaffel, L. (2008). The relationship between the shape of the mental number line and familiarity with numbers in 5- to 9-year old children: Evidence for a segmented linear model. Journal of Experimental Child Psychology, 99, 1-17. doi:10.1016/j.jecp.2007.08.006.

Ebersbach, M., \& Resing, W. C. M. (2008). Implicit and explicit knowledge of linear and exponential growth in 5- and 9-year-olds. Journal of Cognition and Development, 9, 286-309. doi:10.1080/ 15248370802247962 .

Lieven Verschaffel. Centre for Instructional Psychology and Technology, KU Leuven, Dekenstraat 2, box 3773, 3000 Leuven, Belgium.

Current themes of research:

Psychology of mathematics education. Number sense. Estimation. Mental and written arithmetic. Arithmetic word problem solving. Rational number knowledge.

Most relevant publications in the field of Psychology of Education:

Verschaffel, L., Luwel, K., Torbeyns, J., \& Van Dooren, W. (2009). Conceptualizing, investigating, and enhancing adaptive expertise in elementary mathematics education. European Journal of Psychology of Education, 24, 335-359.

Dewolf, T., Van Dooren, W., \& Verschaffel, L. (2011). Upper elementary school children's understanding and solution of a quantitative word problem inside and outside the mathematics class. Learning and Instruction, $21,770-780$.

Fernández, C., Llinares, S., Van Dooren, W., De Bock, D., Verschaffel, L. (2012). The development of students' use of additive and proportional methods along primary and secondary school. European Journal of Psychology of Education, 27, 421-438.

Obersteiner, A., Van Dooren, W., Van Hoof, J., \& Verschaffel, L. (2013). The natural number bias and magnitude representation in fraction comparison by expert mathematicians. Learning and Instruction, 28, 64-72.

Linsen, S., Verschaffel, L., Reynvoet, B., \& De Smedt, B. (2015). The association between numerical magnitude processing and mental versus algorithmic multi-digit subtraction in children. Learning and Instruction, 35, 4250 . 
Koen Luwel. Centre for Instructional Psychology and Technology, KU Leuven, Dekenstraat 2, box 3773, 3000 Leuven, Belgium; Centre for Educational Research and Development, KU Leuven-Campus Brussels, Warmoesberg 26, 1000 Brussels, Belgium.

Current themes of research:

Strategy choice and strategy development. Estimation skills. Numerical and mathematical cognition. Number sense.

Most relevant publications in the field of Psychology of Education:

Luwel, K., Foustana, A., Papadatos, Y., \& Verschaffel, L. (2011). The role of intelligence and feedback in children's strategy competence. Journal of Experimental Child Psychology, 108, 61-76.

Verschaffel, L., Luwel, K., Torbeyns, J., \& Van Dooren, W. (2009). Conceptualizing, investigating, and enhancing adaptive expertise in elementary mathematics education. European Journal of Psychology of Education, 24, 335-359.

Luwel, K., \& Verschaffel, L. (2008). Estimation of 'real' numerosities in elementary school children. European Journal of Psychology of Education, 23, 319-338.

Ebersbach, M., Luwel, K., Frick, A., Onghena, P., \& Verschaffel, L. (2008). The relationship between the shape of the mental number line and familiarity with numbers in 5- to 9-year old children: Evidence for a segmented linear model. Journal of Experimental Child Psychology, 99, 1-17.

Luwel, K., Siegler, R. S., \& Verschaffel, L. (2008). A microgenetic study of insightful problem solving. Journal of Experimental Child Psychology, 99, 210-232. 